\title{
Modelling and Forecasting Unbiased Extreme Value Volatility Estimator: A Study Based on EUR/USD Exchange Rate
}

\author{
Dilip Kumar \\ Indian Institute of Management, Kashipur, India \\ Email: dksic212@gmail.com
}

How to cite this paper: Kumar, D. (2018) Modelling and Forecasting Unbiased Extreme Value Volatility Estimator: A Study Based on EUR/USD Exchange Rate. Theoretical Economics Letters, 8, 1599-1613. https://doi.org/10.4236/tel.2018.89102

Received: March 9, 2018

Accepted: June 10, 2018

Published: June 13, 2018

Copyright $\odot 2018$ by author and Scientific Research Publishing Inc. This work is licensed under the Creative Commons Attribution International License (CC BY 4.0).

http://creativecommons.org/licenses/by/4.0/

\begin{abstract}
The paper provides a framework to model and forecast volatility of EUR/USD exchange rate based on the unbiased AddRS estimator as proposed by Kumar and Maheswaran [1]. The framework is based on the heterogeneous autoregressive (HAR) model to capture the heterogeneity in a market and to account for long memory in data. The results indicate that the framework based on the unbiased extreme value volatility estimator generates more accurate forecasts of daily volatility in comparison to alternative volatility models.
\end{abstract}

\section{Keywords}

Volatility Modeling, Volatility Forecasting, Forecast Evaluation, Economic Significance Analysis, Bias-Corrected Extreme Value Estimator

\section{Introduction}

The volatility of the financial market has implications towards asset pricing, portfolio and fund management and in risk measurement and management. Moreover, more accurate prediction of volatility is important for option valuation, in implementing successful trading strategies and in construction of the optimal hedge using futures. Another important application of volatility is in the estimation and forecasting of Value-at-Risk and expected shortfall.

There are various ways to estimate daily volatility and it depends on the kind of data used. The squared return and the absolute return are the very basic estimates of daily volatility based on close-to-close prices. However, these estimates of daily volatility are highly inefficient in nature [2]. The GARCH class of models uses the squared return as a measure of unconditional volatility in order to generate conditional volatility estimates and forecasts. The daily realized volatil- 
ity is yet another popular estimate of daily volatility and is estimated by using intraday high-frequency data. The realized volatility is estimated by taking sum of squared high-frequency intraday returns. However, the intraday high-frequency data are suffered from various market microstructure issues, which makes its estimation complex. These market microstructure issues can make the realized volatility estimator highly biased. Moreover, high-frequency data are difficult to obtain and are usually expensive and sometime may not be available or may be available only for a shorter duration for various tradable assets. The high-frequency data demands substantial computational resources and time which may not be of interest to the practicing quant [3].

The third popular way of estimating daily volatility makes use of the daily opening, high, low and closing prices. These include the method of moments estimators [1] [4] [5] [6] [7] [8] and maximum likelihood (ML) estimators [9] [10] [11]. These volatility estimators are highly efficient when compared with the close-to-close return based volatility estimators. Out of all these, the Rogers and Satchell [6] (RS) volatility estimator stands out as it is unbiased regardless of the drift. Kumar and Maheswaran [1] highlight the presence of downward bias in the RS estimator and propose a bias corrected version of the RS estimator, referred as the AddRS estimator.

The main contribution of the paper is to propose the use of heterogeneous autoregressive (HAR) model for the AddRS estimator. The model is named as HAR-AddRS model. The HAR-AddRS model can capture the long memory characteristics and heterogeneity in the markets. We also examine the statistical and distributional properties of the AddRS and Log (AddRS) estimator and obtain similar inference as given in the findings of Kumar and Maheswaran [12]. We also implement the HAR-AddRS-GARCH model to account for the heteroskedasticity in the residuals of the HAR-AddRS model. We evaluate the forecasting performance of the AddRS based models using the error statistics approach and the superior predictive ability (SPA) approach and compare the results with the corresponding results based on alternative returns based and range based volatility models. Our findings indicate that the AddRS based models perform much better than the alternative models in forecasting realized volatility.

The remainder of this paper is organized as follows. Section 2 presents the literature review. Section 3 describes the data, methodology and preliminary analysis. Section 4 reports our empirical findings, discussion and policy implications. Section 5 concludes with a summary of our main findings.

\section{Literature Review}

The Generalized Autoregressive Conditional Heteroskedasticity (GARCH) family of models and stochastic volatility models are highly popular in capturing the dynamics of the return based volatility [13] [14]. The GARCH family of models has origin due to the seminal study by Engle [13] and Bollerslev [14]. Since then, these have become the standard models for estimating and forecasting volatility. 
Subsequently, various extensions of GARCH models have been proposed in the literature. Engle and Bollerslev [15] propose the Integrated GARCH (IGARCH) model to capture the impact of a shock on the future volatility over an infinite horizon. Baillie, Bollerslev [16] propose the fractionally integrated GARCH (FIGARCH) model to allow for fractional orders I(d) of integration, where $0<\mathrm{d}$ $<1$. The EGARCH model [17], GJR-GARCH model [18] and APARCH model [19] are popular asymmetric models from the GARCH family. Bollerslev and Ole Mikkelsen [20] fractionally integrated exponential GARCH (FIEGARCH) model and Tse [21] fractionally integrated asymmetric power ARCH (FIAPARCH) model help us incorporate long memory effect with asymmetry in volatility. The literature related to stochastic volatility models started with the work of Clark [22] and Taylor [23]. These models assume that the evolution of volatility over time is an independent stochastic process and also that past returns do not impact future volatility.

Alizadeh, Brandt [2] propose the use of two-factor stochastic volatility model to model range based volatility measures and find that the range based volatility models are highly efficient than the returns based counterparts. Chou [24] proposes the Conditional Autoregressive Range Model (CARR) to model the dynamics in the range-based volatility measures. Chou [24] also provides the extension of the CARR model which include the exogenous variables and named that model as CARRX model to predict volatilities. Chou [24] finds that the CARR model better predicts the volatility in comparison to the GARCH based models. Brandt and Jones [25] suggest the use of exponential generalized autoregressive conditional heteroskedasticity (EGARCH) model with trading range to generate more accurate forecasts of the trading range. Chen, Gerlach [26] provide another extension of the CARR model which include the threshold conditional heteroskedastic autoregressive model. Chen, Gerlach [26] find the presence of significant threshold non-linearity in the data. Chiang and Wang [27] propose the logarithm conditional autoregressive range based model with lognormal distribution to capture the smooth transition in the range process. Li and Hong [28] suggest the use of auto-regression model for range based volatility. Chan, Lam [29] propose the CARR model based on the geometric framework and named that model as the conditional autoregressive geometric process range (CARGPR) model to allows for flexible trend patterns, threshold effects, leverage effects, and long-memory dynamics in financial time series. Kumar and Maheswaran [12] propose the use of ARFIMA based model to generate forecasts based on AddRS estimator. All these studies imply the fact that the forecasts of volatility based on range based volatility estimator are more accurate when compared with the forecasts based on the returns based volatility models. Kumar [30] incorporate the impact of structural breaks in the CARR model while modelling and predicting the RS estimator. Kumar [31] incorporate the impact of structural breaks in modelling the Yang and Zhang [8] volatility estimator. In another study, Kumar [32] incorporate the impact of structural breaks in the CARR model to capture the dynamics of the range based volatility estimator. 
Kumar [33] make use of ARFIMA-AddRS model to model and to generate forecasts of volatility of energy futures. Kumar [34] incorporate the impact of structural breaks in modelling and forecasting the AddRS estimator and find that the model which incorporate the impact of structural breaks in modelling and forecasting volatility provide more accurate forecasts of realized volatility than the return based volatility models. In another study, Kumar [35] provide the approach to incorporate leverage effect in the model to model and predict AddRS volatility estimator.

\section{Data, Methodology and Preliminary Analysis}

\subsection{Data}

We use the daily opening, high, low and closing values of the EUR/USD exchange rate. The sample period is from 1 January 1999 to 25 August 2014. The choice of the sample period is based on the introduction of Euro currency. We consider last 1000 observations for out-of-sample forecast evaluation. We make use of realized volatility, based on the sum of squares of 5 minutes intra-day high-frequency returns, as measured volatility for out-of-sample forecast evaluation exercises. All the data have been collected from the Bloomberg database. We have used MATLAB to perform the analysis.

\subsection{Methodology}

\section{Constructing AddRS Estimator}

Kumar and Maheswaran [1] propose the AddRS estimator, which is unbiased regardless of the drift, based on the reflection principle. Suppose $O_{t}, H_{t} L_{t}$ and $C_{t}$ are the opening, high, low and closing prices of an asset on day $t$. Define:

$$
\begin{gathered}
b_{t}=\log \left(\frac{H_{t}}{O_{t}}\right) \\
c_{t}=\log \left(\frac{L_{t}}{O_{t}}\right) \\
x_{t}=\log \left(\frac{C_{t}}{O_{t}}\right)
\end{gathered}
$$

Let $u_{t}=2 b_{t}-x_{t}$ and $v_{t}=2 c_{t}-x_{t}$. Hence, the bias corrected extreme value estimators are given by:

$$
\text { Add } u x=\frac{1}{2}\left(u_{t}^{2}-x_{t}^{2}\right)+x_{t}^{2} \cdot \mathbf{1}_{\left\{b_{t}=0 \text { or } x_{t}=b_{t}\right\}}
$$

and

$$
\text { Add } v x=\frac{1}{2}\left(v_{t}^{2}-x_{t}^{2}\right)+x_{t}^{2} \cdot \mathbf{1}_{\left\{c_{t}=0 \text { or } x_{t}=c_{t}\right\}}
$$

Therefore, the unbiased AddRS estimator, as proposed by Kumar and Maheswaran [1], is given as: 


$$
\operatorname{AddRS}=\frac{1}{2}[A d d u x+A d d v x]
$$

\subsection{Properties of the AddRS Estimator}

In this section, we examine the properties of the AddRS and the Log (AddRS) estimators of EUR/USD exchange rate. Figure 1 presents the plots of the levels of EUR/USD exchange rate. It can be seen that EUR/USD has experienced significant variation in levels around the period of global financial crisis.

Figure 2 presents the plots of AddRS, Log (AddRS), kernel density of AddRS and kernel density of Log (AddRS) of the EUR/USD exchange rate. The EUR/USD exchange rate exhibits higher volatility during the period of global financial crisis (2007-2009). The kernel density of the AddRS estimator of EUR/USD is highly right skewed and this also supports the findings based on the descriptive statistics (see Figure 1). However, the kernel density of the Log (AddRS) is nearly Gaussian. This indicates that the AddRS estimator exhibits approximately log-normal property.

Figure 1 reports the descriptive statistics of the AddRS estimator of EUR/USD exchange rate. The AddRS estimator of EUR/USD exhibits leptokurtic behavior and is right skewed which indicate its non-normal behavior. The skewness and the excess kurtosis for the Log (AddRS) estimator exhibit significant decline in their values and nearly appear to be Gaussian which is in line with the findings based on the kernel density plots.

Table 1 also reports the long memory parameter $(d)$ for the unconditional AddRS estimator and Log (AddRS) estimator based on exact Local Whittle estimator. The Exact Local Whittle estimator is based on the frequency domain representation (expressed by Discrete Fourier Transform (DFT)) of the observed series and is evaluated at $m$ Fourier frequencies from the spectrum's origin. We have set $m=T^{0.5}$ to estimate the fractional differencing parameter $d$. The Exact Local Whittle estimator tests the null hypothesis of short memory in the volatility series against the alternative hypothesis of long memory.

Null Hypothesis, $H_{0}: d=0$, and Alternative Hypothesis, $H_{1}: d>0$

It can be seen that the fractional integration parameter $(d)$ estimates are significantly greater than zero for both the AddRS estimator and the Log (AddRS) estimator. The $d<0.5$ indicate that the AddRS estimator and the Log (AddRS) estimator follow a covariance stationary process.

\subsection{Heterogeneous Autoregressive AddRS (HAR-AddRS) Model}

Corsi [36] proposed the heterogeneous autoregressive (HAR) model based on the Heterogeneous Market Hypothesis. The HAR model can approximately capture the heterogeneity in the market and long memory in the given time series. The statistical properties of the Log (AddRS) also suggest the use of a linear Gaussian model which can capture the long memory characteristics of the time series. The heterogeneity in the market is considered to be due to the presence of 
various market participants with short- (daily), medium- (weekly) and long-term (monthly) investment horizons.

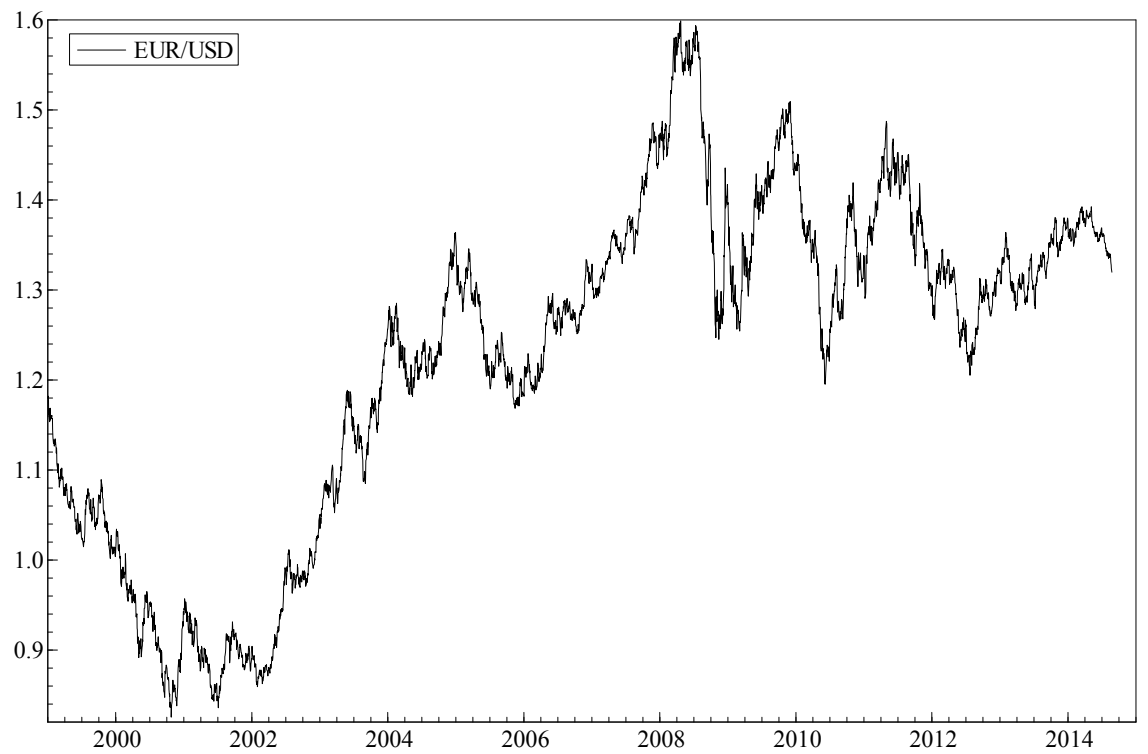

Figure 1. Plot of levels of EUR/USD exchange rate. The EUR/USD has experienced significant variation in levels around the period of global financial crisis.
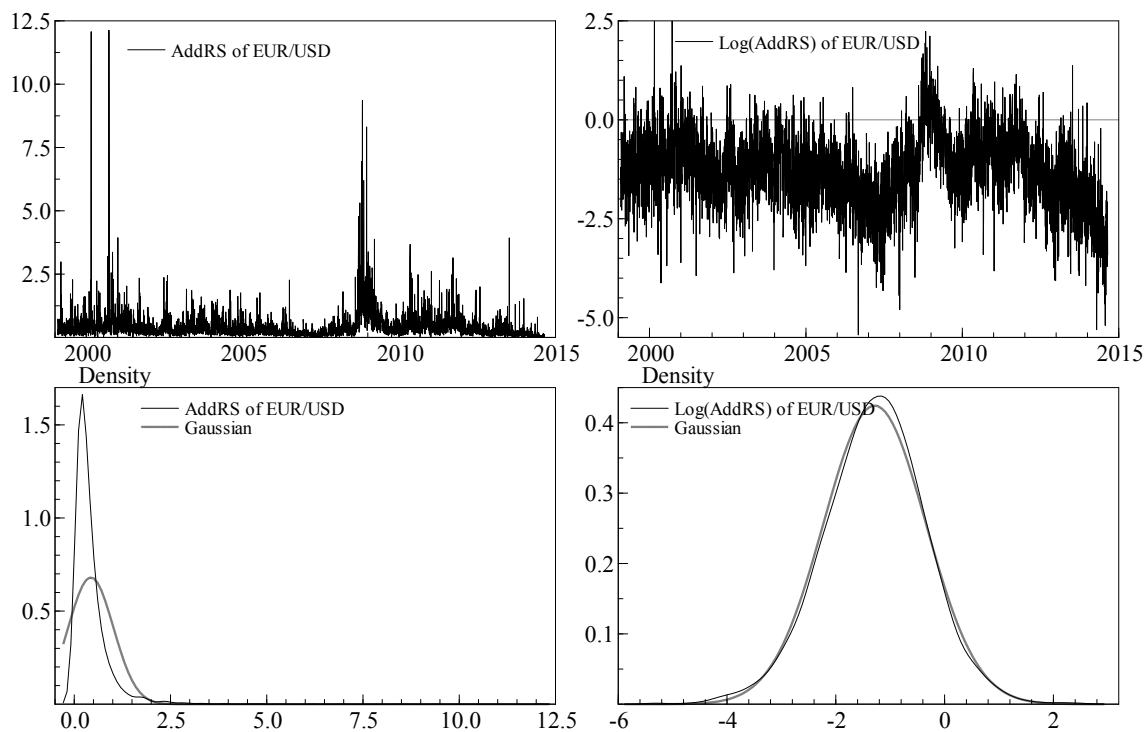

Figure 2. Plots of AddRS, Log (AddRS), the density of AddRS and density of Log (AddRS) for EUR/USD.

Table 1. Descriptive statistics of the daily AddRS and Log (AddRS) estimator of EUR/USD.

\begin{tabular}{ccccccc}
\hline & Mean & Std dev & Skewness & Kurtosis & Q(20) & $d$ \\
\hline AddRS & 0.430 & 0.586 & $8.269^{\#}$ & $120.130^{\#}$ & $6069.820^{\#}$ & $0.237^{\#}$ \\
$\log$ (AddRS) & -1.286 & 0.940 & $-0.135^{\#}$ & $0.607^{\#}$ & $12953.200^{\#}$ & $0.241^{\#}$ \\
\hline
\end{tabular}

$\#$ and $\dagger$ mean significant at $1 \%$ and $10 \%$ level of significance. The estimates of the fractional differencing parameter, $\mathrm{d}$, are based on the Exact Local Whittle estimator. 
The HAR model for the $\log (\operatorname{AddRS})$ is given as:

$$
\begin{aligned}
\log (\operatorname{AddRS})_{t}^{(d)}= & \alpha_{0}+\alpha_{d} \log (\operatorname{AddRS})_{t-1}^{(d)}+\alpha_{w} \log (\operatorname{AddRS})_{t-1}^{(w)} \\
& +\alpha_{m} \log (\operatorname{AddRS})_{t-1}^{(m)}+\varepsilon_{t}
\end{aligned}
$$

where $\log (\operatorname{AddRS})_{t-1}^{(d)}$ is the lagged daily $\log ($ AddRS) estimator, $\log (\operatorname{AddRS})_{t-1}^{(w)}=\frac{1}{5} \sum_{i=1}^{5} \log (\operatorname{AddRS})_{t-i}^{(d)}$ is the lagged weekly volatility component and $\log (\operatorname{AddRS})_{t-1}^{(m)}=\frac{1}{22} \sum_{i=1}^{22} \log (\operatorname{AddRS})_{t-i}^{(d)}$ is the lagged monthly volatility component.

\subsection{Heterogeneous Autoregressive AddRS Generalized Autoregressive Conditional Heteroskedasticity (HAR-AddRS-GARCH) Model}

To account for the conditional heteroskedasticity in the residuals from the HAR-AddRS model, we implement the $\operatorname{GARCH}(1,1)$ error process. The proposed model is given as:

$$
\begin{aligned}
\log (\operatorname{AddRS})_{t}^{(d)}= & \alpha_{0}+\alpha_{d} \log (\operatorname{AddRS})_{t-1}^{(d)}+\alpha_{w} \log (\operatorname{AddRS})_{t-1}^{(w)} \\
& +\alpha_{m} \log (\operatorname{AddRS})_{t-1}^{(m)}+\varepsilon_{t} \\
& \varepsilon_{t}=\sigma_{t} Z_{t}, Z_{t} \sim N(0,1) \\
& \sigma_{t}^{2}=\omega+\alpha_{1} \varepsilon_{t-1}^{2}+\beta_{1} \sigma_{t-1}^{2}
\end{aligned}
$$

\section{Empirical Results}

\subsection{In Sample Analysis}

We implement the HAR model using Log (AddRS) estimator and refer the model as HAR-AddRS model. To capture the heteroskedasticity in the residuals of the HAR-AddRS model, we also implement the heterogeneous autoregressive AddRS generalized autoregressive conditional heteroskedasticity (HAR-AddRS-GARCH) model. We use the Schwarz information criterion (SIC) to select the appropriate orders of the HAR-AddRS-GARCH(p, q) model for Log (AddRS). The orders that minimize the SIC is an HAR-AddRS-GARCH $(1,1)$ specification.

For both the HAR-AddRS model and the HAR-AddRS-GARCH model, the coefficients of lagged weekly $\left(\alpha_{w}\right)$ and monthly $\left(\alpha_{m}\right)$ volatility components are significant at $1 \%$ level of significance indicating that the lagged weekly and monthly volatility components have the greatest impact on the current volatility of the given exchange rates. For the HAR-AddRS model, the lagged daily $\left(\alpha_{d}\right)$ volatility component is significant at $10 \%$ level of significance. On the other hand, for HAR-AddRS-GARCH model, the lagged daily $\left(\alpha_{d}\right)$ volatility component is significant at $1 \%$ level of significance. For both models, the lagged daily volatility has a negative impact on the current volatility of EUR/USD. This indicates that the next day volatility of the given exchange rates is an aggregate effect of short term, medium term and long term volatility components. We also do 
not find any autocorrelation in the squared residuals of the HAR-AddRS-GARCH model based on the Ljung Box statistic up to 20 lags (Tables 2-3).

\subsection{Out-of-Sample Forecast Evaluation}

This section addresses the forecasting performance of the HAR-AddRS and the HAR-AddRS-GARCH models in predicting realized volatility. The results are compared with the corresponding forecasts of the range based conditional autoregressive range model (CARR) and return-based models which include the GARCH, the GJR-GARCH, the EGARCH, the FIGARCH, the FIEGARCH and the RiskMetrics models. We consider the forecasting horizon of 1 day for all the models. We generate 1000 forecasts of 1-day horizon based on the parameter estimates using rolling windows with fixed window size. We generate the realized volatility measure based on 5 minutes high-frequency return data as a proxy for measured volatility $\left(M V_{t}\right)$. Suppose $r_{t, n}$ represents the return for the period from $(n-1)$ to $n$ on day $t$. The daily realized volatility based on 5 minutes returns is given as:

$$
R V_{t}=\sum_{i=1}^{j} r_{t, i}^{2}
$$

Table 2. Parameter estimates and diagnostics for the HAR model.

\begin{tabular}{cc}
\hline & EUR/USD \\
\hline$\alpha_{0}$ & $-0.122^{*}$ \\
$\alpha_{d}$ & $(0.027)$ \\
& $-0.032^{\dagger}$ \\
$\alpha_{w}$ & $(0.019)$ \\
& $0.258^{*}$ \\
$\alpha_{m}$ & $(0.040)$ \\
& $0.681^{*}$ \\
$\mathrm{R}^{2}$ & $(0.039)$ \\
Skewness & 0.372 \\
Kurtosis & -0.084 \\
JB Stat & 3.842 \\
& $124.629^{*}$ \\
Q(20) & {$[0.000]$} \\
& $84.775^{*}$ \\
ARCH(10) & {$[0.000]$} \\
& 1.521 \\
& {$[0.125]$}
\end{tabular}

$\#,{ }^{*}$ and $\dagger$ mean significant at $1 \%, 5 \%$ and $10 \%$ levels of significance, respectively. The terms in parenthesis (.) represent the standard error and the terms in the square braces [.] represent p-value. The JB Stat represents the Jarque Bera statistic, Q(20) represents the Ljung-Box test statistic upto 20 lags and $\mathrm{ARCH}(10)$ represents the ARCH Lagrange Multiplier test statistic up to 10 lags. 
Table 3. Parameter estimates and diagnostics for the HAR-GARCH model.

\begin{tabular}{|c|c|}
\hline & EUR/USD \\
\hline \multirow[t]{2}{*}{$\alpha_{0}$} & $-0.146^{\#}$ \\
\hline & $(0.031)$ \\
\hline \multirow[t]{2}{*}{$\alpha_{d}$} & $-0.068^{\#}$ \\
\hline & $(0.023)$ \\
\hline \multirow[t]{2}{*}{$\alpha_{w}$} & $0.340^{\#}$ \\
\hline & $(0.048)$ \\
\hline \multirow[t]{2}{*}{$\alpha_{m}$} & $0.607^{\#}$ \\
\hline & $(0.046)$ \\
\hline \multirow[t]{2}{*}{$\omega$} & $0.164^{\dagger}$ \\
\hline & $(0.091)$ \\
\hline \multirow[t]{2}{*}{$\alpha$} & $0.035^{*}$ \\
\hline & $(0.018)$ \\
\hline \multirow[t]{2}{*}{$\beta$} & $0.667^{\#}$ \\
\hline & $(0.171)$ \\
\hline$L L$ & -3423.049 \\
\hline AIC & 2.245 \\
\hline BIC & 2.259 \\
\hline \multirow[t]{2}{*}{ JB Stat } & $97.293^{\#}$ \\
\hline & {$[0.000]$} \\
\hline \multirow[t]{2}{*}{$\mathrm{Q}(20)$} & $51.979^{\#}$ \\
\hline & {$[0.000]$} \\
\hline \multirow[t]{2}{*}{ Qs(20) } & 21.641 \\
\hline & {$[0.248]$} \\
\hline \multirow[t]{2}{*}{$\operatorname{ARCH}(10)$} & 0.842 \\
\hline & {$[0.588]$} \\
\hline
\end{tabular}

\#, * and $\dagger$ mean significant at $1 \%, 5 \%$ and $10 \%$ levels of significance, respectively. The terms in parenthesis (.) represent the standard error and the terms in the square braces [.] represent p-value. The JB Stat represents the Jarque Bera statistic, Q(20) represents the Ljung-Box test statistic upto 20 lags and $\mathrm{ARCH}(10)$ represents the ARCH Lagrange Multiplier test statistic up to 10 lags.

where $j$ depends on the number of 5 minutes returns in a day. We undertake statistical forecast evaluation exercises based on the error statistics test and superior predictive ability test to test the forecasting performance of the HAR-AddRS and the HAR-AddRS-GARCH models.

\subsubsection{Out-of-Sample Forecast Evaluation Based on Error Statistic (Loss Functions)}

We use the following five loss functions for evaluating the forecasting performance of the models. 
1) Mean squared errors (MSE)

$$
\operatorname{MSE}(m, h)=\frac{1}{T} \sum_{t=1}^{T}\left(F V_{t+h}(m)-M V_{t+h}\right)^{2}
$$

2) Mean absolute errors $(M A E)$

$$
\operatorname{MAE}(m, h)=\frac{1}{T} \sum_{t=1}^{T}\left|F V_{t+h}(m)-M V_{t+h}\right|
$$

3) Heteroskedasticity adjusted mean square error (HMSE)

$$
\operatorname{HMSE}(m, h)=\frac{1}{T} \sum_{t=1}^{T}\left(1-\frac{M V_{t+h}}{F V_{t+h}(m)}\right)^{2}
$$

4) Heteroskedasticity adjusted mean absolute error (HMAE)

$$
\operatorname{HMAE}(m, h)=\frac{1}{T} \sum_{t=1}^{T}\left|1-\frac{M V_{t+h}}{F V_{t+h}(m)}\right|
$$

5) Logarithmic loss function $(L L)$

$$
L L(m, h)=\frac{1}{T} \sum_{t=1}^{T}\left(\ln \left(\frac{M V_{t+h}}{F V_{t+h}(m)}\right)\right)^{2}
$$

where $m$ represents the models under consideration, $h$ can be related to the $h$-step(s) ahead forecasts, $M V_{t}$ represents the measured volatility at time $t$ (realized volatility at time $t), F V_{t}(m)$ represents the volatility forecast made by the model $m$ and $T$ represents the number of out-of-sample volatility forecasts. Here, $h$ is taken to be 1 and $T$ is taken to be 1000 .

Table 4 reports the results based on loss functions to evaluate the out-of-sample forecasting performance of the models under study. The HAR-AddRS model outperforms the alternative models by providing the minimum value of all the loss functions with few exceptions. The GJR-GARCH model and the RiskMetrics model perform equally by providing the same lowest value of the LL loss function. Overall, the HAR-AddRS model performs much better than the alternative models in providing more accurate forecasts of realized volatility based on the loss functions. The HAR-AddRS-GARCH model ranks second in providing the lowest values of the loss functions.

\subsubsection{Multiple Comparison Test for out-of-Sample Forecast Evaluation} We also make use of Hansen [37] superior predictive ability test (hereafter referred as SPA test) to compare the performance of the HAR-AddRS and the HAR-AddRS-GARCH models with alternative models in forecasting realized volatility.

\section{1) Hansen's [37] superior predictive ability test}

Table 5 reports the $p$-values of SPA test based on MSE, MAE, HMSE, HMAE and LL error statistics. Results indicate that when HAR-AddRS model is a benchmark model then we cannot reject the null hypothesis of the superior predictive ability of the benchmark model for all error statistics at 5\% level of significance. 
Table 4. Out-of-sample forecast evaluation based on loss functions for EUR/USD.

\begin{tabular}{ccccccccccc}
\hline & HAR & HAR-G & CARR & GARCH & GJR-GARCH & EGARCH & FIGARCH & FIEGARCH & Risk-Metrics \\
\hline$M S E$ & 0.031 & 0.032 & 0.312 & 0.033 & 0.033 & 0.033 & 0.037 & 0.034 & 0.033 \\
$M A E$ & 0.113 & 0.115 & 0.532 & 0.118 & 0.118 & 0.117 & 0.123 & 0.120 & 0.114 \\
$H M S E$ & 0.345 & 0.361 & 14.611 & 0.650 & 0.661 & 0.640 & 0.672 & 0.705 & 0.525 \\
$H M A E$ & 0.361 & 0.370 & 2.800 & 0.462 & 0.462 & 0.461 & 0.461 & 0.473 & 0.408 \\
LL & 0.245 & 0.266 & 1.678 & 0.247 & 0.245 & 0.246 & 0.265 & 0.257 & 0.245 \\
\hline
\end{tabular}

Where HAR and HAR-G represent the HAR-AddRS model and the HAR-AddRS-GARCH model.

Table 5. Out-of-sample forecast evaluation based on SPA test.

\begin{tabular}{ccccccccccc}
\hline & HAR & HAR-G & CARR & GARCH & GJR-GARCH & EGARCH & FIGARCH & FIEGARCH & Risk-Metrics \\
\hline EUR/USD & & & & & & & & & & \\
MSE & 1.000 & 0.000 & 0.000 & 0.207 & 0.599 & 0.525 & 0.006 & 0.028 & 0.458 \\
MAE & 1.000 & 0.000 & 0.000 & 0.274 & 0.328 & 0.387 & 0.027 & 0.024 & 0.682 \\
$H M S E$ & 1.000 & 0.004 & 0.000 & 0.013 & 0.028 & 0.007 & 0.037 & 0.017 & 0.026 \\
$H M A E$ & 1.000 & 0.000 & 0.000 & 0.002 & 0.002 & 0.000 & 0.002 & 0.000 & 0.022 \\
LL & 0.935 & 0.000 & 0.000 & 0.463 & 0.857 & 0.787 & 0.007 & 0.009 & 1.000 \\
\hline
\end{tabular}

Where HAR and HAR-G represent the HAR-AddRS model and the HAR-AddRS-GARCH model.

For the HAR-AddRS-GARCH, CARR, FIGARCH and FIEGARCH models acting as a benchmark model, the null hypothesis is rejected for all the loss functions. The GARCH, GJR-GARCH, EGARCH and RiskMetrics models exhibit an equal number of successes ( 3 out of 5). Overall, it can be seen that the HAR-AddRS model outperforms other models in generating superior forecasts of realized volatility.

\subsection{Policy Implications}

Predicting volatility in exchange rate more accurately has implications for regulators, traders, market makers, fund managers and financial institutions. The continuous presence of high volatility in a market with a decline in levels can signal for the forthcoming crisis and recession. This can help the regulators in implementing appropriate strategies to stabilize the economy. The trader can implement profitable trading strategies by generating more accurate forecasts of volatility to take out better short-term gains. For financial institutions and fund managers, the precise forecasts of exchange rate volatility can help in pricing derivatives securities and in portfolio rebalancing at the appropriate time. Hedgers can mitigate risk by estimating appropriate hedge ratio based on more accurate forecasts of volatility. The portfolios of institutional investors usually contain a variety of derivatives. Moreover, options are traded in terms of the volatility of the underlying asset. Furthermore, the more accurate forecast of the exchange rate volatility has important implications for investors, portfolio managers and 
risk managers having off-shore exposure. Volatility plays an important role in portfolio theory and helps in optimal allocation of investors' money in different asset classes or securities. The choice of the optimal portfolio is usually based on minimizing the risk (measured by volatility). Another important implication of the findings is the estimation and forecasting of value-at-risk (VaR) and Expected Shortfall. The Basle Committee on Banking Supervision has recommended the compulsory implementation of risk management practices in financial institutions around the world. Financial institutions, regulators, business practitioners and portfolio managers use $\mathrm{VaR}$ as a measure of market risk. It helps the financial institutions to determine the minimum capital to deal with catastrophic event in the market.

\section{Conclusion}

In this paper, we propose to use a simple HAR model-based framework to model and to generate more accurate volatility forecasts based on the AddRS estimator. We also propose to use GARCH specification with HAR-AddRS model to capture heteroscedasticity in the AddRS volatility series using HAR-AddRS-GARCH model. To evaluate the out-of-sample forecasting performance of the proposed framework, we make use of realized volatility, based on high-frequency data, as a measure of actual volatility. The statistical and distributional properties of the AddRS and Log (AddRS) estimators also support the use of the linear Gaussian model to model them. The findings based on the in-sample analysis support the evidence of better fit by HAR-AddRS model for Log (AddRS). We evaluate the out-of-sample forecasting performance of the HAR-AddRS and HAR-AddRS-GARCH models using error statistic approach and the SPA test. We make use of five loss functions (MSE, MAE, HMSE, HMAE and LL) for the error statistic approach. The findings indicate that the HAR-AddRS model outperforms the alternative models in generating more accurate forecasts of realized volatility by providing lowest value for all error statistics. The results based on the SPA tests also confirm the similar findings that the HAR based models outperform return based and range based volatility models in generating superior forecasts of volatility. The study does not incorporate the impact of structural breaks, leverage effect and jumps in volatility while modelling and generating forecasts of the same. Further research can be undertaken by incorporating the impact of structural breaks, leverage effect and jumps in volatility.

\section{References}

[1] Kumar, D. and Maheswaran, S. (2014) A Reflection Principle for a Random Walk with Implications for Volatility Estimation Using Extreme Values of Asset Prices. Economic Modelling, 38, 33-44. https://doi.org/10.1016/j.econmod.2013.11.045

[2] Alizadeh, S., Brandt, M.W. and Diebold, F.X. (2002) Range-Based Estimation of Stochastic Volatility Models. The Journal of Finance, 57, 1047-1091.

https://doi.org/10.1111/1540-6261.00454

[3] Rogers, L.C. and Zhou, F. (2008) Estimating Correlation from High, Low, Opening 
and Closing Prices. The Annals of Applied Probability, 18, 813-823. https://doi.org/10.1214/07-AAP460

[4] Parkinson, M. (1980) The Extreme Value Method for Estimating the Variance of the Rate of Return. Journal of Business, 53, 61-65. https://doi.org/10.1086/296071

[5] Garman, M.B. and Klass, M.J. (1980) On the Estimation of Security Price Volatilities from Historical Data. The Journal of Business, 53, 67-78. https://doi.org/10.1086/296072

[6] Rogers, L.C. and Satchell, S.E. (1991) Estimating Variance from High, Low and Closing Prices. The Annals of Applied Probability, 1, 504-512.

https://doi.org/10.1214/aoap/1177005835

[7] Kunitomo, N. (1992) Improving the Parkinson Method of Estimating Security Price Volatilities. The Journal of Business, 65, 295-302. https://doi.org/10.1086/296570

[8] Yang, D. and Zhang, Q. (2000) Drift-Independent Volatility Estimation Based on High, Low, Open, and Close Prices. The Journal of Business, 73, 477-492.

https://doi.org/10.1086/209650

[9] Ball, C.A. and Torous, W.N. (1984) The Maximum Likelihood Estimation of Security Price Volatility: Theory, Evidence, and Application to Option Pricing. The Journal of Business, 57, 97-112. https://doi.org/10.1086/296226

[10] Magdon-Ismail, M. and Atiya, A.F. (2003) A Maximum Likelihood Approach to Volatility Estimation for a Brownian Motion Using High, Low and Close Price Data. Quantitative Finance, 3, 376-384. https://doi.org/10.1088/1469-7688/3/5/304

[11] Horst, E.T., et al. (2012) Stochastic Volatility Models Including Open, Close, High and Low Prices. Quantitative Finance, 12, 199-212. https://doi.org/10.1080/14697688.2010.492233

[12] Kumar, D. and Maheswaran, S. (2014) Modeling and Forecasting the Additive Bias Corrected Extreme Value Volatility Estimator. International Review of Financial Analysis, 34, 166-176. https://doi.org/10.1016/j.irfa.2014.06.002

[13] Engle, R.F. (1982) Autoregressive Conditional Heteroscedasticity with Estimates of the Variance of United Kingdom Inflation. Econometrica: Journal of the Econometric Society, 50, 987-1007. https://doi.org/10.2307/1912773

[14] Bollerslev, T. (1986) Generalized Autoregressive Conditional Heteroskedasticity. Journal of Econometrics, 31, 307-327. https://doi.org/10.1016/0304-4076(86)90063-1

[15] Engle, R.F. and Bollerslev, T. (1986) Modelling the Persistence of Conditional Variances. Econometric Reviews, 5, 1-50. https://doi.org/10.1080/07474938608800095

[16] Baillie, R.T., Bollerslev, T. and Mikkelsen, H.O. (1996) Fractionally Integrated Generalized Autoregressive Conditional Heteroskedasticity. Journal of Econometrics, 74, 3-30. https://doi.org/10.1016/S0304-4076(95)01749-6

[17] Nelson, D.B. (1991) Conditional Heteroskedasticity in Asset Returns: A New Approach. Econometrica: Journal of the Econometric Society, 59, 347-370. https://doi.org/10.2307/2938260

[18] Glosten, L.R., Jagannathan, R. and Runkle, D.E. (1993) On the Relation between the Expected Value and the Volatility of the Nominal Excess Return on Stocks. The Journal of Finance, 48, 1779-1801. https://doi.org/10.1111/j.1540-6261.1993.tb05128.x

[19] Ding, Z., Granger, C.W. and Engle, R.F. (1993) A Long Memory Property of Stock Market Returns and a New Model. Journal of Empirical Finance, 1, 83-106. 
https://doi.org/10.1016/0927-5398(93)90006-D

[20] Bollerslev, T. and Ole Mikkelsen, H. (1996) Modeling and Pricing Long Memory in Stock Market Volatility. Journal of Econometrics, 73, 151-184.

https://doi.org/10.1016/0304-4076(95)01736-4

[21] Tse, Y.K. (1998) The Conditional Heteroscedasticity of the Yen-Dollar Exchange Rate. Journal of Applied Econometrics, 13, 49-55. https://doi.org/10.1002/(SICI)1099-1255(199801/02)13:1<49::AID-JAE459>3.0.CO; $2-\mathrm{O}$

[22] Clark, P.K. (1973) A Subordinated Stochastic Process Model with Finite Variance for Speculative Prices. Econometrica: Journal of the Econometric Society, 41, 135-155. https://doi.org/10.2307/1913889

[23] Taylor, S.J. (1986) Modelling Financial Time Series. 2nd Edition, John Wiley and Sons, Hoboken.

[24] Chou, R.Y. (2005) Forecasting Financial Volatilities with Extreme Values: The Conditional Autoregressive Range (CARR) Model. Journal of Money, Credit and Banking, 37, 561-582. https://doi.org/10.1353/mcb.2005.0027

[25] Brandt, M.W. and Jones, C.S. (2006) Volatility Forecasting with Range-Based EGARCH Models. Journal of Business \& Economic Statistics, 24, 470-486. https://doi.org/10.1198/073500106000000206

[26] Chen, C.W., Gerlach, R. and Lin, E.M. (2008) Volatility Forecasting Using Threshold Heteroskedastic Models of the Intra-Day Range. Computational Statistics \& Data Analysis, 52, 2990-3010. https://doi.org/10.1016/j.csda.2007.08.002

[27] Chiang, M.-H. and Wang, L.-M. (2011) Volatility Contagion: A Range-Based Volatility Approach. Journal of Econometrics, 165, 175-189.

https://doi.org/10.1016/j.jeconom.2011.07.004

[28] Li, H. and Hong, Y. (2011) Financial Volatility Forecasting with Range-Based Autoregressive Volatility Model. Finance Research Letters, 8, 69-76. https://doi.org/10.1016/j.frl.2010.12.002

[29] Chan, J.S., et al. (2012) A Bayesian Conditional Autoregressive Geometric Process Model for Range Data. Computational Statistics \& Data Analysis, 56, 3006-3019. https://doi.org/10.1016/j.csda.2011.01.006

[30] Kumar, D. (2015) Sudden Changes in Extreme Value Volatility Estimator: Modeling and Forecasting with Economic Significance Analysis. Economic Modelling, 49, 354-371. https://doi.org/10.1016/j.econmod.2015.05.001

[31] Kumar, D. (2016) Sudden Breaks in Drift-Independent Volatility Estimator Based on Multiple Periods Open, High, Low, and Close Prices. IIMB Management Review, 28, 31-42. https://doi.org/10.1016/j.iimb.2016.02.001

[32] Kumar, D. (2016) Sudden Changes in Crude Oil Price Volatility: An Application of Extreme Value Volatility Estimator. American Journal of Finance and Accounting, 4, 215-234. https://doi.org/10.1504/AJFA.2016.080717

[33] Kumar, D. (2017) Forecasting Energy Futures Volatility Based on the Unbiased Extreme Value Volatility Estimator. IIMB Management Review, 29, 294-310. https://doi.org/10.1016/j.iimb.2017.11.002

[34] Kumar, D. (2017) Structural Breaks in Unbiased Volatility Estimator: Modeling and Forecasting. Journal of Prediction Markets, 11, 399-421.

[35] Kumar, D. (2017) Modeling and Forecasting Unbiased Extreme Value Volatility Estimator in Presence of Leverage Effect. Journal of Quantitative Economics, 1-23. https://doi.org/10.1007/s40953-017-0085-4 
[36] Corsi, F. (2009) A Simple Approximate Long-Memory Model of Realized Volatility. Journal of Financial Econometrics, 7, 174-196.

https://doi.org/10.1093/jifinec/nbp001

[37] Hansen, P.R. (2005) A Test for Superior Predictive Ability. Journal of Business \& Economic Statistics, 23, 365-380. https://doi.org/10.1198/073500105000000063 